\title{
Ground Truth Design Principles
}

\author{
An Overview \\ Daniel Kondermann \\ Heidelberg Collaboratory for Image Processing \\ Speyerer Strasse 16 \\ 69115 Heidelberg, Germany \\ daniel.kondermann@iwr.uni-heidelberg.de
}

\begin{abstract}
Ground truth or reference data is the basis for performance analysis in computer vision and image processing. This paper summarizes design considerations for ground truth acquisition with a focus on accuracy and cost of different approaches. I argue that first and foremost, requirements engineering needs to be carried out and it needs to be decided whether quantitative performance analysis is beneficial to the field of research at all. Three categories of reference data are presented. Examples for each approach are given and advantages and downsides discussed. Finally, open issues are briefly summarized.
\end{abstract}

\section{Categories and Subject Descriptors}

B.8.0 [Performance and Reliability]: General-Ground Truth; C.4 [Performance of Systems]: Measurement techniques; D.2.4 [Software Engineering]: Software/Program Verification-Reliability, Validation; D.2.8 [Software Engineering]: Metrics-Performance Measures; I.3.0 [Computer Graphics]: General; I.4.0 [Image Processing and Computer Vision]: General

\section{General Terms}

Ground Truth, Design Principles, Performance Analysis, Benchmarking, Reference Data

\section{INTRODUCTION}

We are drowning in algorithms, but starving for insights: after three decades of innovation and exploration in methodological computer vision research we now face the challenges of consolidating the findings to date. Literally thousands of algorithms have been published in each sub-discipline such as optical flow, segmentation, denoising, etc. Engineers as well as high-level vision researchers have to choose "the right" algorithms for their given problem domains. As a result, performance analysis and benchmarking as a means

Permission to make digital or hard copies of all or part of this work for personal or classroom use is granted without fee provided that copies are not made or distributed for profit or commercial advantage and that copies bear this notice and the full citation on the first page. To copy otherwise, to republish, to post on servers or to redistribute to lists, requires prior specific permission and/or a fee.

VIGTA '13 July 15 2013, St. Petersburg, Russia

Copyright 2013 ACM 978-1-4503-2169-3/13/07 ...\$10.00. of ranking different techniques is currently becoming a hot topic in research.

A common assumption is that the basis for performance analysis is ground truth generation. In this short paper, I argue that the actual first step for any performance analysis is requirements engineering: only with well known problem definitions we can choose to create the right kind of ground truth. I also argue that in many occasions ground truth generation is not necessary for performance analysis. As the most important constraints are the accuracy of the data, the cost of and the time needed for acquisition as well as the quantity of data to be recorded, it is often not feasible to e.g. focus on large quantities of highly accurate ground truth. Therefore, I introduce the concept of three types of reference data. Finally, I will give an outlook on other relevant topics associated with performance analysis.

\section{REQUIREMENTS ENGINEERING}

The aim of performance analysis is to understand under which circumstances a given algorithm is suitable for a given task. Very often, computer vision aims at generalization, i.e. solving a large set of challenges with a single algorithm. For example, the task of optical flow estimation is loosely defined as finding a displacement vector for each pixel in an image sequence.

We can ask two questions: what is an image sequence? And why would we want to find the flow at each pixel?

The simplest answer to the first question could be that it is a series of images taken with a camera. Yet, this entails many more questions: what kind of camera was used? Was it a consumer photo camera? A digital SLR? An X-Ray imager? A multispectral camera? A time of flight camera? Is the sensor a rolling-shutter CMOS device? Did we use a wide-angle quartz glass lens which is transparent for ultraviolet light? Based on the answer we might ask for the settings of the camera: what was the frame rate? Which exposure time did we use? What about gain, focal length, aperture, etc.? Now one might guess that the image content might also play a role. Did we record a sports event, dolphins in the sea, a visualization of fluid motion, satellite or airborne images, the human brain, heart or stomach? Is it reasonable to assume that there is one algorithm to "rule" all of these image sequences?

The second question (why to compute dense or sparse flow) is closely related: in some scenarios such as frame interpolation the dense flow requirement makes sense, in others (such as particle image velocimetry) it might even be counter-productive. 
If we consider computer vision to be an engineering science striving for building a generic set of theories and tools to deal with visual data effectively, the first step in algorithm design is a very clear understanding of the requirements. Requirements engineering is a well-studied field of research (cf. e.g. [7]) but rarely applied to computer vision. The choice of content for ground truth datasets can in my opinion only be answered by first developing enabling technologies for ground truth acquisition.

So once the application scenario is clear, requirements engineering can begin. An example is a driver assistance system that operates the brakes in emergency situations. A rough outline of the requirements could be that it runs in real time, is robust under adverse light and weather conditions, consumes little energy while running on embedded hardware, is self-aware about its confidence of estimates and degrades gracefully (meaning that with smoothly increasing adversity of conditions, the system output quality smoothly decreases as well).

Some of these requirements (e.g. robustness, graceful degradation) have to be defined very carefully: what exactly are the adverse conditions that can occur?

But do we actually need quantitative performance evaluations? This question is very interesting and should be asked before ground truth is generated. Generally, the answer depends on whether computer vision is applied either to safety relevant systems such as driver assistance, medical imaging or algorithms which are designed to be measurement tasks such as fluid flow estimation for aerodynamics analysis or wheather forecasting. We do not need ground truth for a proof of concept of an exotic idea or a new class of algorithm. Here, visual analysis of a small dataset is sufficient to motivate further research. We also do not need ground truth for purely aesthetic or artistic methods, even though perceptual studies might be a viable approach to compare results.

Only at this point can we start thinking about a ground truth dataset. It should contain a representative sample of all relevant image sequences that will occur in the real world. One implication of this property is that ideally the data was acquired by the same camera system with the same parameters. Another implication is the open question of which data we need - and how much of it.

This position paper gives an abstract overview of existing techniques. I will focus on low-level vision. It is not the aim of this paper to give a report on the state of the art of concrete methods. Instead, I want to emphasize the properties and design options of ground truth acquistion techniques.

\section{THREE TYPES OF REFERENCE DATA}

The term ground truth originally stems from geography where information drawn from satellite images is confirmed by people visiting the location to be studied on the ground. In medical imaging, the term gold standard often is used to refer to the best available ground truth given reasonable assumptions.

One can also see computer vision as a measurement science where cameras along with algorithms can be understood as measurement devices. In this context, the question for random and systematic deviations from the true values arises. This is usually evaluated with respect to a device whose accuracy is well-known and at least one order of magnitude higher than that of the device to be evaluated. The data generated by such a device is referred to as reference data.

For many computer vision tasks such as optial flow estimation no such device exists. To still be able to analyse the performance of vision systems, I am going to relax the term reference data by creating three sub-categories:

- Reference data without ground truth is a collection of datasets without any knowledge about the results the vision system should yield.

- Reference data with weak ground truth is a collection of datasets including an estimate of the wanted results, but the accuracy of this information is either unknown or less than a magnitude more accurate than the system to be evaluated.

- Finally, reference data with ground truth is a dataset including the wanted results which are at least one order of magnitude more accurate than the quality that can be expected from the vision system at hand.

In the following, several approaches for the generation of each type of reference data will be discussed exemplarily. For brevity, I will focus on my research results only. References to related works are contained within the cited papers.

\subsection{Reference Data Without Ground Truth}

On first sight, just recording a lot of data might not seem like a reasonable approach to do performance analysis, but in my opinion this approach has been underrated in the past. For example, in [9], we recorded around $15 \mathrm{~TB}$ of high-quality stereo image sequences in a driver assistance scenario. In this case, actually creating any kind of ground truth for such highly dynamic outdoor scenes at different times of year and day and varying weather conditions is currently impossible. Yet, driver assistance systems need to be evaluated with respect to their safety under real-world conditions. One obvious advantage of reference data without ground truth is that very large quantities can be collected without too much user interaction. This enables us to sample from reality as densely as possible at relatively low cost and helps to understand the statistics of the application domain. Another advantage is that we can ask questions such as whether we have collected enough data regardless of the financial and technical constraints of ground truth generation.

When it comes to evaluation, very often engineers intuitively understand situations where their systems might fail and relevant or interesting scenarios can be selected and inspected by hand. This process, often referred to as eyeballing can be a viable source of inspiration for further algorithmic developments.

On the other hand a downside of this approach is that an evaluation cannot be carried out fully automatically and is subject to dataset bias due to human interpretation.

The ECCV 2012 Robust Vision Challenge ${ }^{1}$ was based on such a dataset. Researchers were invited to submit their results on a subset of the data aquired in [9]. The results were analysed by an international jury consisting of resarchers and engineers working in application domains such as automotive systems and entertainment. Noteably, all eight jury members concordantly voted for the same winner.

${ }^{1}$ http://hci.iwr.uni-heidelberg.de/Static/challenge2012/ 


\subsection{Reference Data With Weak Ground Truth}

Although reference data without grund truth can be helpful in performance analysis, it is severely limited due to the need for experts. Financially, we trade in acquisition time for eyeballing time which cannot be automated.

This tradeoff can be mitigated by finding methods to annotate existing reference data with ground truth information while ignoring any hard accuracy constraints. Evaluation results have to be taken with a grain of salt, but gross errors, biases or regularly occuring problems (such as occlusion boundaries) can be identified automatically at a large scale. The main question about weak ground truth is how accurate can we become without spending too much time and money?

\subsubsection{The "More Data" Approach}

More data can be used to generate ground truth fully automatically. For example for optical flow, we can use very high quality cameras with high resolution, low noise and high frame rates and use existing flow methods on the generated images. The results as well as the images can then be downsampled, virtually increasing the accuracy. Additional modalities or sensors such as inertial measurement units, global positioning systems, camera arrays, light field cameras, infrared structured light sources and time of flight cameras can be used to increase the amount of information.

Two main drawbacks of this approach are apparent. First, we need to engineer systems that exploit the information from all devices and few methods already exist. Second, we have to obtain knowledge about the accuracy of the system which can be difficult as we need to bootstrap from existing, well-studied measurement technology. Finally, another problem is that such a system can be very costly or only applicable to specific scenarios such as laboratory setups.

One example for such a system are the Middlebury benchmarks. Its optical flow dataset [1] was created by adding UV paint to the scene in order to generate an invisible texture that can be seen only with UV light being turned on. High resolution cameras and a stop motion technique were used to acquire images both in the visible spectrum as well as under UV light. A blockmatching optical flow method was applied to the hidden texture images and the results were downsampled. The estimated accuracy of this approach is approximately $\frac{1}{60}$ of a pixel. Yet, the confidence of this estimate has not been validated by a method with higher accuracy and each flow vector lacks an error bar. Another example is the KITTI dataset[4] were a high-speed LIDAR system along with an inertial measurement unit were utilized.

Creating ground truth with this approach is restricted to small scales and very time consuming but the setup is very cost-effective. This results in large costs for personell and a high entry-barrier to produce new datasets.

Another example is the usage of the KinectFusion system[5] as it is implemented in the current version of the Kinect $\mathrm{SDK}^{2}$ to quickly generate $3 \mathrm{D}$ scans of a scene and use camera position information to project $3 \mathrm{D}$ motion to $2 \mathrm{D}$ flow fields. We studied the accuracy of this approach in [8] and found that it is around 5 to $10 \mathrm{~mm}$ accurate in most cases and shows very little bias. Given a reasonable distance of the camera to the scene, we can therefore achieve subpixel

\footnotetext{
${ }^{2}$ http://www.microsoft.com/en-us/kinectforwindows/
}

accurate flow fields and stereo disparity maps.

The advantage of this method is that it is both low-cost as well as easy to use without consuming much time. The disadvantage of this method is that we need to constrain the scene to be static and relatively small-scale (around 3 cubic meters).

\subsubsection{Annotations and Semiautomatic Ground Truth}

Another option to generate weak ground truth is to ask humans for annotations. In machine learning and high-level vision this is a common practice as annotations usually do not have to be subpixel accurate.

For other tasks, such as dense correspondences or video matting this problem becomes more difficult, as we need to ask questions or create user interfaces that facilitate the process and avoid asking for annotations at each pixel location.

We studied this problem with respect to optical flow in [3] which is based on the first work with this idea conducted by [6]. Here, we asked crowdworkers to annotate contours and feature tracks of planar surfaces and compared the quality to existing ground truth datasets and results obtained by experts. The results are ecouraging as we were able to obtain subpixel accurate results in most regions, while bias and object contours can degrade the quality significantly. The method works very well with piecewise planar dynamic scenes, but becomes less accurate with curved surfaces and highly cluttered objects such as vegetation.

This approach scales well due to crowdsourcing, but the average cost per frame of around $\$ 10$ to $\$ 40$ still prohibits the generation of very large amounts of data. Furthermore, humans introduce unknown biases which cannot easily be analysed or predicted systematically.

\subsection{Reference Data With Ground Truth}

Finally, whenever money and technology are available in sufficient quantities, ground truth can be generated with accuracies well beyond the expected quality of the vision system to be evaluated. The most important design consideration here is whether real data should be recorded or computer generated datasets are to be synthesized.

\subsubsection{Ground Truth from Measurement Devices}

Real data with ground truth can only be acquired with measurement devices with very high accuracy and precision. In the case of dense correspondences, structured light 3D scanners are known to be the most accurate devices. Depending on measurement volume and technology, scans can be as accurate as 5 microns.

The main problems with 3D scanners are usually that they produce huge amounts of data that need to be manually processed in most cases. Scanning the office with a LIDAR system and the statue with a structured light scanner in [8] each took around half a day. Furthermore, these devices often cost well above $\$ 100 \mathrm{k}$ and have to be operated by educated staff which has to be paid as well. Another problem are depth edges and shiny or translucent materials which generally create unknown systematic errors. These are usually clearly visible and can be filtered by humans (which again have to be paid for their work).

When 3D scans have been acquired, many geometry- and correspondence-based vision algorithms can be evaluated in case the camera location is known very accurately. This can be achieved by established camera-tracking devices based 
e.g. on reflective markers and appropriate hand-eye calibrations.

For other algorithms, such as video matting, outdoor human motion capturing, material classification, large scale structure from motion, etc. such technologies might not be sufficient. Generally, engineering systems for real ground truth generation is financially not feasible.

\subsubsection{Synthetic Reference Data}

Computer graphics have been used early on for ground truth generation, but have often been considered to be too unrealistic. With the advent of high-end graphic systems e.g. for entertainment this view is currently changing. A recent example is [2]. However, we have to be clear that it remains a yet unproven hypothesis whether creating synthetic images is realistic enough or not. We undertook a first endeavour into understanding how vision algorithms react to graphics in contrast to real images in [10]. The idea was to create a real and a synthetic scene with the same ground truth and to see whether the optical flow measured on both scenes is identical ${ }^{3}$. This project focused on a single algorithm and a single scene. However, the results were promising in that higher degrees of realism in the graphics system showed to yield more similar results to the real scene. On the other hand, the spatial distribution of flow differences revealed some systematic differences caused e.g. by texture boundaries, anti-aliasing material properties.

The most relevant advantage of creating synthetic data is that it can be systematically parameterized: given a scene, the amount of motion, the time of day, weather, material properties and any kind of sensor or lens degradations can be simulated.

Next to the fact that physically correct (in contrast to visually appealing) rendering is less well-studied, an important downside is that the creation of large amounts of data requires highly educated artists and similar amounts of time and money when compared to the previously discussed measurement device approach.

\section{CONCLUSION}

In this article, I have scratched the surface of ground truth generation for low-level vision. I consider dense correspondence ground truth to be the most difficult to create and some considerations might not be applicable to other algorithms which can be evaluated in a more straightforward manner. It would be interesting to see scientists from other fields in computer vision addressing this issue to broaden the perspective for this eventually emerging field of research.

Summarizing, there are two important steps in ground truth generation: First, requirements engineering need to be carried out to find out which type of data is needed. There is almost no research on how this process can be carried out in a systematic and rigorous manner for computer vision. We also do not know when we have enough data for a given task or how to compare existing datasets with respect to their applicability to new tasks.

Second, cost is the main factor. Existing datasets such as Middlebury[1], MPI-Sintel[2], KITTI[4] and HCI[9] range in the hundreds of thousands of dollars when including cost for personell and hardware ${ }^{4}$. Another question is how the

${ }^{3}$ We call it the Turing Test for vision algorithms

${ }^{4}$ According to the authors of the respective datasets, the approach scales with larger amounts of hardware: a system might be expensive when first designed and implemeted but data acquisition might be easy and quick afterwards. In case accuracy is not of utmost importance, this is where the most money can be saved.

Designing systems or methodologies for reference data acquisition is a young field of research at the intersection of science and engineering and puts an interesting emphasis on the measurement science point of view. In my research, I focus on the design of systems everyone can use to create her or his own datasets. Future topics should include the question on how to test whether the requirements are met based on ground truth.

\section{REFERENCES}

[1] S. Baker, D. Scharstein, J. P. Lewis, S. Roth, M. J. Black, and R. Szeliski. A database and evaluation methodology for optical flow. International Journal of Computer Vision, 92(1):1-31, 2011.

[2] D. J. Butler, J. Wulff, G. B. Stanley, and M. J. Black. A naturalistic open source movie for optical flow evaluation. In A. Fitzgibbon et al. (Eds.), editor, European Conf. on Computer Vision (ECCV), Part IV, LNCS 7577, pages 611-625. Springer-Verlag, Oct. 2012.

[3] A. Donath and D. Kondermann. Is crowdsourcing for optical flow ground truth generation feasible? Proc. International Conference on Vision Systems, 2013.

[4] A. Geiger, P. Lenz, and R. Urtasun. Are we ready for autonomous driving? the kitti vision benchmark suite. In Computer Vision and Pattern Recognition (CVPR), Providence, USA, June 2012.

[5] S. Izadi, R. Newcombe, D. Kim, O. Hilliges, D. Molyneaux, S. Hodges, P. Kohli, J. Shotton, A. Davison, and A. Fitzgibbon. Kinectfusion: real-time dynamic $3 \mathrm{~d}$ surface reconstruction and interaction. In ACM SIGGRAPH 2011 Talks, page 23. ACM, 2011.

[6] C. Liu, W. T. Freeman, E. H. Adelson, and Y. Weiss. Human-assisted motion annotation. IEEE Computer Society Conference on Computer Vision and Pattern Recognition, (CVPR08), 0:1-8, 2008.

[7] L. Maciaszek. Requirements analysis and system design. Pearson Education, 2007.

[8] S. Meister, S. Izadi, P. Kohli, M. Hämmerle, C. Rother, and D. Kondermann. When can we use kinectfusion for ground truth acquisition? Proc. Workshop on Color-Depth Camera Fusion in Robotics, 2012.

[9] S. Meister, B. Jähne, and D. Kondermann. Outdoor stereo camera system for the generation of real-world benchmark data sets. Optical Engineering, 51, 2012.

[10] S. Meister and D. Kondermann. Real versus realistically rendered scenes for optical flow evaluation. In Proceedings of 14 th ITG Conference on Electronic Media Technology. Informatik Centrum Dortmund e.V., 2011.

estimated costs are around $\$ 48 \mathrm{k}$ for the Middlebury flow dataset, $\$ 38 \mathrm{k}$ for MPI-Sintel, $\$ 513 \mathrm{k}$ for KITTI and $\$ 270 \mathrm{k}$ for $\mathrm{HCI}$ including both hardware as well as personell. 